\section{Microcystic stromal tumour arising in association with serous cystadenoma}

Sir,

Microcystic stromal tumour (MST) is a rare ovarian stromal neoplasm initially described by Irving and Young in $2009^{1}$ and characterised morphologically by a triad of microcysts, solid cellular areas and hyalinised fibrous stroma. These elements were present in variable proportion in individual cases and some tumours showed a predominant solid growth pattern. The cells typically demonstrate epithelioid morphology with bland cytological appearances and infrequent mitotic activity, but focal degenerative 'symplastic' nuclear atypia may be seen as in other ovarian sex cord stromal tumours (SCSTs). Recently, the histological spectrum of MST has been expanded to include cases lacking these archetypal features, and such cases are likely to present greater diagnostic difficulty. ${ }^{2,3}$ Nevertheless, accurate diagnosis is important in view of the generally benign clinical behaviour of MST and its occasional association with familial adenomatous polyposis (FAP). ${ }^{4-6}$ Herein we describe a further case of 'variant' MST that presented as a solid nodule within the wall of an ovarian serous cystadenoma.

A 57-year-old nulliparous woman presented with intermittent left sided abdominal pain. She had a past medical history of diabetes, hypertension and hysterectomy for benign reasons. An ultrasound scan showed a $4 \mathrm{~cm}$ complex but non-sinister appearing ovarian cyst with a normal serum CA125. Examination revealed mild tenderness in the left pelvis. Surgery was delayed due to COVID restrictions and a repeat ultrasound 3 months later showed a marginal increase in size of the ovarian lesion. The patient underwent uneventful laparoscopic bilateral salpingo-oophorectomy.

The ovaries were up to $35 \mathrm{~mm}$ and $32 \mathrm{~mm}$ in size and both showed multicystic cut surface appearances with a $9 \mathrm{~mm}$ solid pale nodule in one ovary. Histologically, the cysts were lined by benign serous epithelium. The nodule was composed of a lobulated to sheet-like arrangement of plump epithelioid cells with moderate pale to eosinophilic cytoplasm (Fig. 1). The cells displayed round to oval nuclei, often with small but prominent nucleoli, and there were rare nuclear grooves. Occasional cells showed spindled appearances and delicate blood vessels were present. Focal cytoplasmic vacuolation was present but there was no distinct microcystic component and stromal hyalinisation was not observed. The tumour abutted the serous-lined cysts but did not involve the ovarian capsular surface. The fallopian tubes were normal grossly and microscopically.

The initial differential diagnosis of the nodular tumour included luteinised adult granulosa cell tumour (AGCT), thecoma, steroid cell tumour, solid pseudopapillary neoplasm (SPN), and MST. Immunohistochemistry demonstrated diffuse cytoplasmic CD10 expression and nuclear steroidogenic factor 1 (SF1), Wilms tumour 1 gene product (WT1), cyclin D1 and beta-catenin (nucleocytoplasmic) staining (Fig. 2). There was no expression of calretinin, inhibin, actin, desmin, oestrogen receptor (ER), progesterone receptor (PR), human melanoma black 45 (HMB45) or neural cell adhesion molecule (CD56). Reticulin encircled individual cells. The overall findings were consistent with the diagnosis of 'solid' (non-microcystic) variant MST.

Although rare, MST is not likely to present diagnostic difficulty when demonstrating typical histological features. However, McCluggage and colleagues recently presented four cases of MST that demonstrated variant morphology including corded, nested or tubular architectural patterns with limited or absent microcysts; one tumour showed focal spindled cell appearances. ${ }^{2}$ A further case of entirely solid MST was included amongst a series of diagnostically problematic ovarian SCSTs that were subject to molecular analysis. ${ }^{3}$ In these morphologically unusual cases the final diagnosis of MST was supported by the characteristic immunophenotype including CD10, WT1, SF1, beta-catenin (nucleocytoplasmic) and cyclin D1 positivity, and the lack of expression of ER/PR inhibin and calretinin. Furthermore, the four cases that underwent molecular analysis demonstrated alterations in CTNNB1 (beta-catenin) which appears to be the most common genetic alteration in these tumours, with a minority of cases exhibiting mutually exclusive mutations in $A P C .^{6-8}$

As noted above, the initial differential diagnosis considered in the current case included a number of other ovarian neoplasms mainly within the sex cord stromal category and importantly including potentially malignant neoplasms such as AGCT. Most of these could be excluded based upon the immunohistological findings but one tumour worthy of specific consideration is SPN which usually presents in the pancreas but rarely arises in the ovary. ${ }^{9,10}$ Microcystic stromal tumour and SPN share morphological and
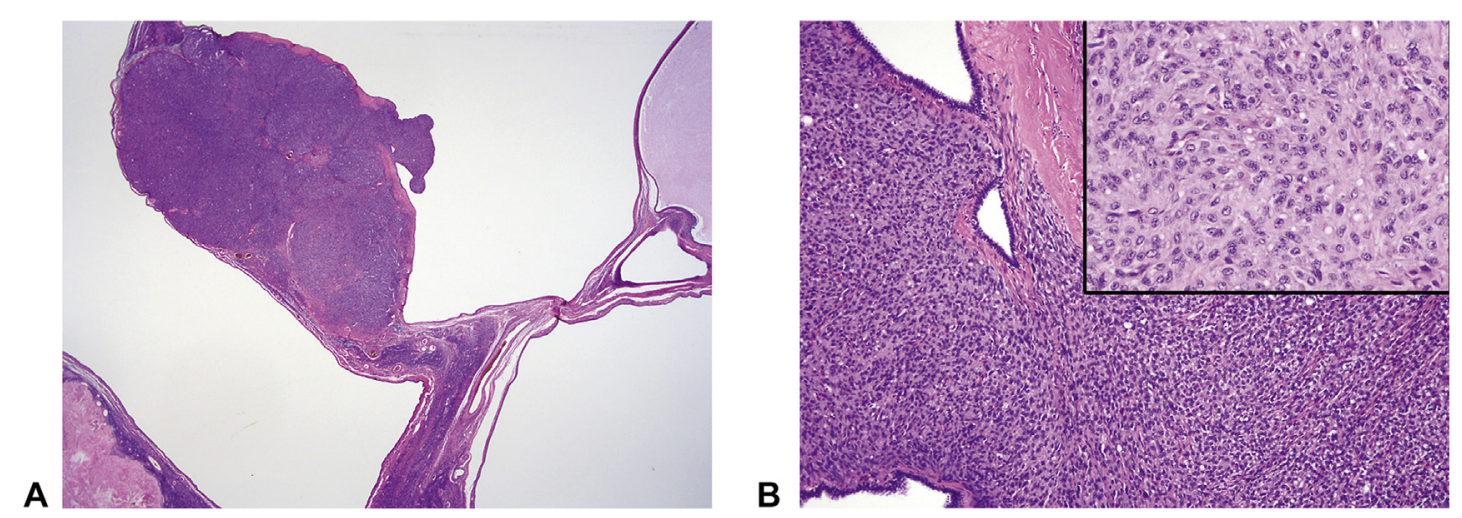

Fig. 1 (A) Histological features at low power showing MST arising in serous cystadenoma and (B) higher power with inset displaying solid and spindled architecture. 
A
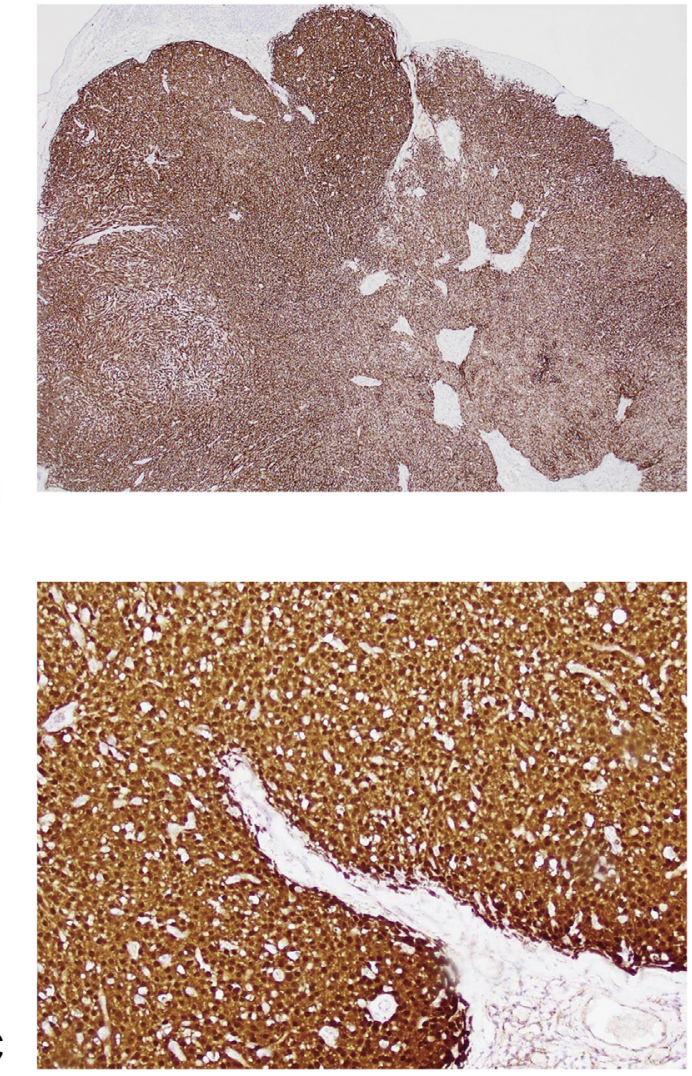

B

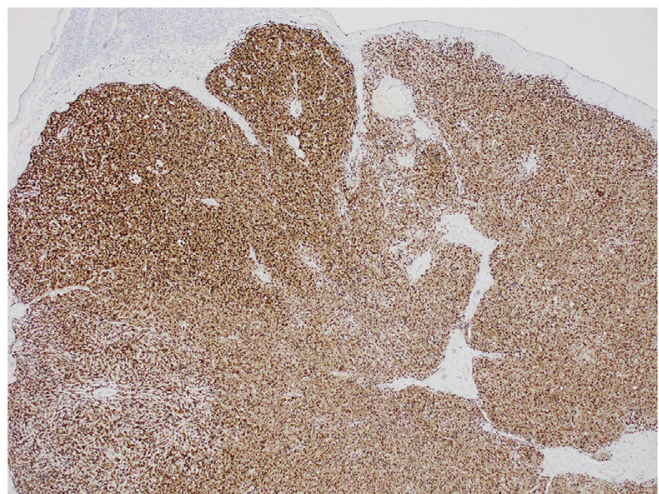

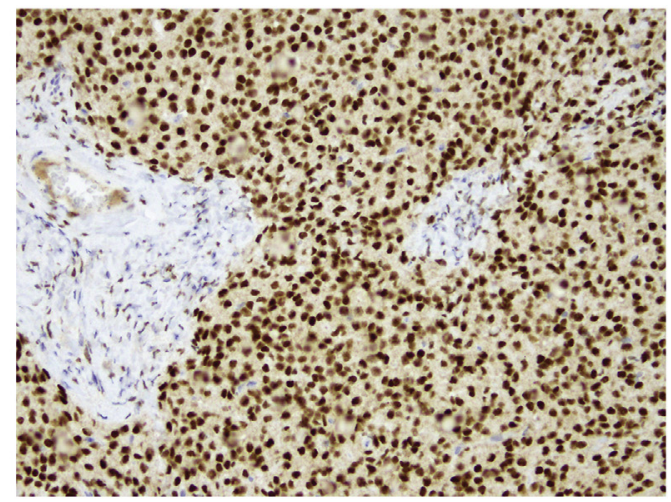

Fig. 2 Immunohistochemistry. (A) CD10 and (B) cyclin D1 at low power. (C) Beta-catenin and (D) SF1 at high power

immunohistochemical features (CD10, cyclin D1 and nucleocytoplasmic beta-catenin staining). However, unlike SPN, MST typically demonstrates nuclear WT1, SF1 and FOXL2 expression with negative CD56, CD99 and PR staining.

The present case was also unusual in that the MST presented as a nodule within an otherwise typical ovarian serous cystadenoma, an association that has not been described previously to our knowledge. Possible explanations include the juxtaposition of two pathogenically unrelated neoplasms ('collision tumours'), or heterologous epithelial differentiation within a stromal neoplasm. The latter phenomenon is a well-recognised feature of other ovarian SCSTs, particularly Sertoli-Leydig cell tumour, and has also been reported occasionally in AGCT. ${ }^{11}$ However, in most such cases the epithelium demonstrates mucinous rather than serous differentiation. We believe that the association of MST and serous cystadenoma in our case was most likely coincidental, an interpretation supported by the presence of a serous cystadenoma in the contralateral ovary.

While MST is generally considered benign, there is one reported case of recurrence more than 10 years after initial resection in a 33-year-old patient. ${ }^{12}$ The tumour demonstrated an $A P C$ mutation and the patient also had multiple adenomatous colonic polyps raising the possibility of FAP. Three of four additional MSTs demonstrating APC mutations have been associated with FAP, suggesting that MST is a rare extracolonic manifestation of this condition. ${ }^{4-6,8}$ While molecular analysis was not performed in the case presented here, the patient had no other significant medical history and recent colonoscopy showed no adenomatous polyps.

Conflicts of interest and sources of funding: The authors state that there are no conflicts of interest to disclose.

\section{Yancey Wilson ${ }^{1}$, Stuart Salfinger ${ }^{2}$, Colin Stewart ${ }^{1,3}$}

${ }^{1}$ Clinipath Pathology, Osborne Park, Perth, WA, Australia;

${ }^{2}$ Western Australian Gynae and Surgery, Perth, WA, Australia; ${ }^{3}$ Pathwest, King Edward Memorial Hospital, Subiaco, Perth, WA, Australia

Contact Dr Yancey Wilson.

E-mail: ywilson@ clinipath.net

1. Irving JA, Young RH. Microcystic stromal tumor of the ovary: report of 16 cases of a hitherto uncharacterized distinctive ovarian neoplasm. Am J Surg Pathol 2009; 33: 367-75.

2. McCluggage WG, Chong AS, Attygalle AD, et al. Expanding the morphological spectrum of ovarian microcystic stromal tumour. Histopathology 2019; 74: 443-51.

3. Stewart CJR, Amanuel B, De Kock L, et al. Evaluation of molecular analysis in challenging ovarian sex cord-stromal tumours: a review of 50 cases. Pathology 2020; 52: 686-93.

4. Lee SH, Koh YW, Roh HJ, Cha HJ, Kwon Y-S. Ovarian microcystic stromal tumor: a novel extracolonic tumor in familial adenomatous polyposis. Genes Chromosomes Cancer 2015; 54: 353-60.

5. Liu C, Gallagher RL, Price GR, et al. Ovarian microcystic stroma tumor: a rare clinical manifestation of familial adenomatous polyposis. Int J Gynecol Pathol 2016; 35: 561-5.

6. McCluggage WG, Irving JA, Chong A-S, et al. Ovarian microcystic stromal tumors are characterized by alterations in the beta-catenin-APC 
pathway and may be an extracolonic manifestation of familial adenomatous polyposis. Am J Surg Pathol 2018; 42: 137-9.

7. Irving JA, Lee $\mathrm{C}-\mathrm{H}$, Yip $\mathrm{S}$, et al. Microcystic stromal tumor: a distinctive ovarian sex cord-stromal neoplasm characterized by FOXL2 SF-1, WT-1, Cyclin D1, and beta-catenin nuclear expression and CTNNB1 mutations. Am J Surg Pathol 2015; 39: 1420-6.

8. Rabban JT, Karnezis AN, Devine WP. Practical roles for molecular diagnostic testing in ovarian adult granulosa cell tumour, Sertoli-Leydig cell tumour, microcystic stromal tumour and their mimics. Histopathology 2020; 76: 11-24.

9. Deshpande V, Oliva E, Young RH. Solid pseudopapillary neoplasm of the ovary: a report of 3 primary ovarian tumors resembling those of the pancreas. Am J Surg Pathol 2010; 34: 1514-20.

10. Komforti MK, Edelman M, Fan C, Liang SX. Solid pseudopapillary neoplasm presenting as a primary ovarian mass in an eighteen-year-old female: report of a case and review of the literature. Virchows Arch 2018; 472: 285-91.

11. McKenna M, Kenny B, Dorman G, McCluggage WG. Combined adult granulosa cell tumor and mucinous cystadenoma of the ovary: granulosa cell tumor with heterologous mucinous elements. Int J Gynecol Pathol 2005; 24: 224-7.

12. Zhang Y, Tao L, Yin C, et al. Ovarian microcystic stromal tumor with undetermined potential: case study with molecular analysis and literature review. Hum Pathol 2018; 78: 171-6.

DOI: https://doi.org/10.1016/j.pathol.2021.02.018

\section{Infectious crystalline keratopathy: the clinical utility of periodic acid- Schiff stain}

Sir,

Infectious crystalline keratopathy (ICK) describes a pattern of chronic corneal infection characterised by needle-shaped colonies of microorganisms growing between collagen lamella with disproportionally little inflammation. ${ }^{1}$ The condition arises when microorganisms gain access to the corneal stroma of patients whose local immune response has been suppressed by topical corticosteroids. The majority of infections are caused by low virulence bacteria, but fungi and acanthamoeba have been reported. ${ }^{1}$ We describe a patient with ICK due to periodic acid-Schiff (PAS)-positive bacteria who developed post-operative endophthalmitis more than a month after penetrating keratoplasty. Delayed onset postoperative endophthalmitis is an uncommon but serious complication of intraocular surgery. Knowledge that PASpositive bacteria are putative contaminants in this clinical setting can alter management.

A man in his late eighties underwent a penetrating keratoplasty to restore vision that had declined to 'hand motions' from a failed corneal transplant (Descemet's stripping endothelial automated keratoplasty) 6 years earlier for Fuchs' dystrophy. For 9 months prior to penetrating keratoplasty the patient was treated with topical prednisolone acetate $1 \%$ (varying from hourly to four times per day). During this time the cornea was diffusely oedematous and focally 'cloudy'. Surgery was uncomplicated; no tissue was submitted for culture. The clinicians were alerted days after surgery that the cornea was infected (see below). Topical moxifloxacin, started after surgery, was discontinued on post-operative day 22. Recovery remained uneventful until post-operative day 42 when the patient developed anterior chamber inflammation and elevated intraocular pressure. He was started on topical prednisolone acetate $1 \%$ for non-specific 'uveitis' and pressure lowering medications (dorzolamide 2\%/timolol $0.5 \%$ ). On post-operative day 50, inflammation substantially worsened so oral valtrex was begun on the presumptive diagnosis of herpes uveitis (there was no previous history of ocular herpes infection or any characteristic findings, e.g., epithelial dendrite, etc.). Inflammation worsened until post-operative day 93 at which time cultures from aqueous and vitreous humours were taken; both grew Corynebacterium striatum with greater colony counts from the anterior chamber. Intravitreous antibiotics (ceftazidime $2.25 \mathrm{mg} / 0.1 \mathrm{~mL}$; vancomycin $1 \mathrm{mg} / 0.1 \mathrm{~mL}$ ) were injected the day the samples were obtained; intraocular inflammation progressively improved over several weeks. Final visual acuity was $20 / 400$.

On microscopic examination, the central cornea was superficially ulcerated. Innumerable bacteria were growing in stroma between collagen lamellae forming spindle shaped colonies (Fig. 1). There was modest focal acute inflammation around the outer edge of bacterial colonies. Deeper stroma and Descemet's membrane (earlier graft tissue) were separated from anterior stroma. Descemet's membrane was covered on its posterior surface with fibrin and degenerating neutrophils Gram stain showed mostly Gram-positive cocci and small nonbranching Gram-positive rods (Fig. 1C). The bacteria stained strongly with PAS after diastase and were acid-fast negative with Ziehl-Neelsen and Fite stains. A Gomori methenamine silver (GMS) stain was negative for fungus.

The histopathological findings in this case were diagnostic of ICK, namely the biofilm-like proliferation of bacteria between collagen lamellae with disproportionately little inflammatory response. Secondary endophthalmitis is an anticipated complication after contaminated intraocular surgery, but in this case endophthalmitis was unusual in that the signs of intraocular inflammation were delayed more than a month after surgery. ${ }^{2,3}$ Delayed onset endophthalmitis is a manifestation of a limited subset of low-virulence organisms. The putative bacterial contaminants found in this case were PAS-positive which also limits possible pathogens. PASpositive bacteria include Bacillus cereus, Klebsiella pneunomiae, Micrococcus luteus, Propionibacterium, Corynebacterium, Tropheryma whipplei, Actinomyces, and atypical mycobacteria. ${ }^{4,5}$ In context of this case, GMS and AFB stains along with morphological findings excluded atypical mycobacteria and actinomycetes. Bacillus cereus and Klebsiella pneumonia are highly virulent ocular pathogens and cause rapidly progressive endophthalmitis. ${ }^{6}$ Micrococcus luteus and Tropheryma whipplei have not been implicated in postoperative endophthalmitis. To our knowledge, only two PAS-positive bacteria have been associated with chronic (e.g., delayed onset) endophthalmitis: propionibacterium and corynebacterium. ${ }^{7,8}$

Corynebacterium spp., are Gram-positive bacilli that are part of the normal flora of the skin and eyelid. Most members of this genus are regarded as laboratory contaminants that rarely cause human infection. Corynebacterium striatum, however, may be different. It has been implicated in cases of endocarditis, meningitis, bacteraemia, and respiratory infection. ${ }^{9,10}$ In the context of this case, $C$. striatum appears as both a low grade pathogen and an opportunistic organism that has colonised corneal stroma. The patient had no systemic immune-compromising conditions (other than age) but the structural integrity of his eye was compromised by the complications of past surgery. 